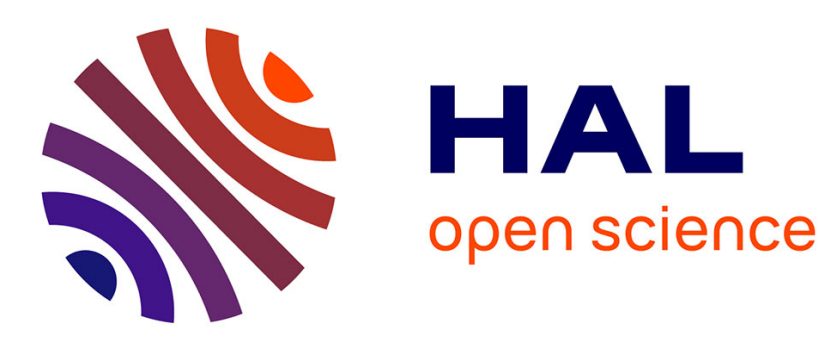

\title{
Le chèque (voucher), instrument néolibéral et/ou innovation institutionnelle ?
}

\author{
Arnaud Lacheret
}

\section{To cite this version:}

Arnaud Lacheret. Le chèque (voucher), instrument néolibéral et/ou innovation institutionnelle ?. Entreprendre \& Innover, 2017, 32, pp.36-50. 10.3917/entin.032.0036 . halshs-01628710

\section{HAL Id: halshs-01628710 \\ https://shs.hal.science/halshs-01628710}

Submitted on 4 Nov 2017

HAL is a multi-disciplinary open access archive for the deposit and dissemination of scientific research documents, whether they are published or not. The documents may come from teaching and research institutions in France or abroad, or from public or private research centers.
L'archive ouverte pluridisciplinaire HAL, est destinée au dépôt et à la diffusion de documents scientifiques de niveau recherche, publiés ou non, émanant des établissements d'enseignement et de recherche français ou étrangers, des laboratoires publics ou privés. 


\section{Le chèque (voucher), instrument néolibéral et/ou innovation institutionnelle?}

$>$ Arnaud Lacheret

\section{Résumé}

Le chèque (ou voucher) est un instrument d'action publique d'origine anglo-saxonne, dont l'usage s'est largement diffusé au sein des collectivités locales françaises. Dans cet article, l'auteur entend démontrer, sur la base d'une étude de terrain menée auprès d'acteurs de collectivités locales, que cette diffusion s'est faite de façon dépolitisée, par le biais de l'action des entreprises qui en assuraient la mise en oeuvre en insistant sur son caractère « innovant ».

(( début du texte))

Le chèque ou «voucher» en anglais, est un instrument d'action publique (Lascoumes, Le Galès, 2004) au statut scientifique paradoxal : objet très étudié dans le monde anglo-saxon, il reste peu analysé en France, alors même que son usage et sa promotion s'y sont largement répandus.

La réinvention et la transformation du chèque en « objet » de science politique peuvent être précisément datés. Milton Friedman, père du monétarisme et penseur du néolibéralisme, va en effet relancer cette approche et tenter de l'appliquer au domaine des services publics et notamment du financement de l'éducation (Friedman, 1955, 1962). II imagine un système déréglementé où les parents seront libres de leur choix éducatif, grâce à l'attribution d'un «voucher » ou chèqueéducation, une forme d'allocation versée directement aux familles, qui leur permettra de choisir l'école dans laquelle ils placeront leurs enfants. Les parents seront libres de dépenser cette somme et d'éventuels compléments dans les services éducatifs de leur choix, lesquels suivront un minimum de standards approuvés et homologués par l'administration fédérale à Washington DC. 
II faudra attendre les années 1990 en France pour que cet instrument, apparu dans un contexte néolibéral largement anglo-américain, devienne un outil incontesté de politique publique en France, un dispositif de compromis efficace pour les parties prenantes de l'action publique et, de facto, une innovation institutionnelle pérenne.

Cet article propose une analyse des fondements idéologiques et pragmatiques du « chèque » et un repérage de sa trajectoire singulière en France pour tenter de déterminer si cette innovation est le produit d'un transfert des politiques publiques menées outre-Atlantique ou s'il s'agit d'une innovation institutionnelle certes inspirée d'un corpus néolibéral influent mais plus sous-jacent, en tout cas facilement acclimaté au contexte national du fait de son apparente neutralité instrumentale.

L'article mobilise, dans une première partie, deux notions clefs - le néolibéralisme en tant qu'idéologie et le chèque en tant qu'instrument avant de procéder ensuite à une analyse discursive des perceptions et des propos des acteurs pouvant expliquer la rapide diffusion et appropriation de ce dernier mais la relative la rémanence de "marqueurs » néolibéraux dans les conceptions que se font les entrepreneurs politiques et les divers publics concernés. La troisième partie conclusive montre que le chèque, pour devenir un outil légitime de l'action publique s'est en fait technicisé et dépolitisé suite au rôle de médiation assuré par les entreprises et les organisations chargées de le mettre en œuvre, gommant ainsi sa dimension idéologique et politique initiale.

\section{Le néolibéralisme anglo-saxon : un contexte d'émergence favorable}

La notion de néolibéralisme rapportée à un instrument, a fortiori au chèque éducation, se nourrit de références plus anciennes que celles de Friedman. On peut 
remonter notamment à John Stuart Mill (1860), qui évoque déjà, pour un contexte anglais, l'importance du choix des parents pour l'éducation que pourraient recevoir leurs enfants. Hayek puis Friedman, sont les promoteurs américains de ce que Michel Foucault définit comme une technique de gouvernement de soi et des autres visant à étendre l'emprise des mécanismes du marché aux individus et à la société tout entière.

Dans sa leçon sur la biopolitique, Foucault indique que dans le néolibéralisme, le marché est d'abord un milieu concurrentiel où l'individu n'est plus simplement une partie de l'échange, mais devient un véritable entrepreneur de lui-même. On peut rapprocher cette idée de ce que le très libéral Ludwig Von Mises évoquait en parlant de l'individu planificateur produisant sa propre planification. Appliquée aux institutions éducatives et aux instruments de gestion, cette conception issue du corpus néolibéral permet d'introduire et d'étendre à d'autres sphères le concept de de quasi-marché (Glennester, Le Grand, 1995). Sont ainsi transférées les logiques du modèle marchand au monde de l'enseignement public dans une perspective de réforme en profondeur l'éducation aux Etats-Unis. La démarche propose une forme de régulation alternative, marchande et individualisée, qui peut suppléer financements étatiques des systèmes éducatifs publics considérés comme trop coûteux. L'octroi d'un chèque/voucher peut ainsi se substituer, pour partie, aux financements éducatifs et servir de modèle de compromis entre des subventions massives publiques et un système totalement privé. C'est dans ce nouveau contexte dérégulé que l'usage du chèque s'est développé.

Dans une perspective néolibérale, il faut donc que la puissance publique n'intervienne que dans le but de permettre à l'individu-entrepreneur d'exercer ses libres choix et de faire que le plan d'action personnel qu'il a retenu se concrétise. 
L'Etat doit donc garantir le bon fonctionnement des mécanismes de marché et permettre à l'individu de sélectionner les offres de formation de son choix. C'est la vision de l'économiste de Chicago, Friedman, lorsque ce dernier conçoit le chèque éducation: l'individu doit avoir la possibilité d'accéder librement au marché de l'éducation grâce à une dotation qu'il peut librement utiliser.

\section{Le chèque : un instrument pragmatique issu de visions néolibérales}

Cette idée va être notamment expérimentée au Chili en 1981 où Friedman ira conseiller le Gouvernement du général Pinochet pour implanter le premier chèque éducation dans les années 80 . II sera décliné sous diverses formes, notamment dans plusieurs Etats américains (comme le Milwaukee et un certain nombre d'Etats républicains comme la Floride, qui utilise toujours le système du "school voucher ») et, notamment, un état-providence européen comme la Suède).

Le «voucher» a d'abord été considéré par les chercheurs anglo-saxons, comme un outil de politique publique «idéologiquement neutre » et pouvant être comparé à n'importe quel élément d'une «boîte à outils » des politiques publiques avant d'être très largement critiqué ${ }^{1}$.

Cette arrivée massive des «outils » et «instruments » dans les politiques publiques dans les années 1990 est qualifiée de «révolution que personne n'a remarquée »(Salamon, 2000). Eugene Steuerle, considère également que le chèque ne constitue qu'un outil parmi d'autres, plus ou moins efficace en fonction du sujet et de la politique publique abordés. Le chèque est avant tout un objet social, visant à fournir une aide sociale ciblée à des bénéficiaires précisément identifiés.

\footnotetext{
${ }^{1}$ La Secrétaire à l'Education nommée par Donald Trump, Betsy DeVos a été très critiquée à cause de son engagement en faveur du chèque devant le Sénat.
} 
Cette notion d'outil pragmatique qui n'a rien d'idéologique pour les chercheurs qui l'étudient (et qui s'en défendent), se retrouve chez d'autres auteurs spécialistes du chèque. Citons Gary Sturgess et Ivana Bodroza, qui évoquent un « dispositif technique, un outil qui aide à bonifier la valeur de services publics quand le marché et les politiques publiques vont dans le même sens ».

Dans un autre ouvrage consacré au chèque, Steuerle définit le « voucher » comme une subvention qui procure un pouvoir d'achat limité à un individu, lequel peut choisir parmi un choix restreint de biens et de services (Steuerle, 2000).

Ultérieurement, de très nombreuses études critiques viendront mettre à mal cette idée d'un chèque « neutre » et mettront en évidence son sens néolibéral tout en en critiquant fortement l'efficacité. Le temps de l'évaluation du chèque (Lacheret, 2014), particulièrement après les premières tentatives dans plusieurs Etats américains mettra cet outil au centre d'une controverse scientifique et en fera un enjeu politique particulièrement important, on citera Martin Carnoy (2008), Wolf (2012), Chingos et Peterson (2013), Feigenberg et al (2017) ainsi que de nombreux articles dans la presse généraliste et spécialisée.

C'est cette définition instrumentale qui semble le mieux rendre compte du succès rencontré par l'adoption du chèque en France, notre objet d'étude, et plus particulièrement du transfert de la conception anglo-saxonne. Nous la préférerons à celle de Harvey Rosen où le « voucher » ne devient qu' « une subvention affectée pour certains besoins, comme les soins médicaux ou l'éducation donnée à des individu» (Rosen, 2001) qui est très utilisée notamment par les auteurs qui cherchent à se défendre de traiter d'un objet considéré comme idéologiquement trop marqué. 
Avec l'extension, en particulier aux Etats-Unis, de la logique du chèque vers d'autres domaines (logement, aides sociales...) et, à la suite d'Eugene Steuerle (2000, 2001), Lester Salamon (2001), Jay Greene (1998, 2001, 2011), Gary Sturgess et Ivana Bodroza (2011), nous avons pu isoler six arguments principaux justifiant dans ce cadre de pensée, la mise en place d'un système de chèque : la promotion du libre-choix pour un public ciblé, la notion d'équité sociale motivant le versement d'une aide à un public ciblé auprès des contribuables ou de la population en général, la promotion d'un principe de mise en concurrence entre fournisseurs de biens et services payés par les chèques, le remplacement par une allocation spécifique d'un programme de financement public existant, l'encadrement et la limitation du choix du public bénéficiaire, et enfin la baisse des coûts de financement des politiques publiques d'aide.

Le Nouveau Management Public (Hood, 1991) a naturellement repris l'instrument en s'appuyant sur une double logique argumentative essentielle : une politique de "voucher » doit être considérée non seulement du point de vue du bénéfice apporté au récipiendaire mais également du point de vue du financeur. Dans ce cas, les notions de «public value» (Moore, 1995) et celle de «merit goods » (Becker, 1974) sont invoquées.

Ainsi, le «voucher » est identifié dans les discours de recherche comme un instrument reposant sur une conception «néolibérale » des politiques publiques et des acteurs individuels, notamment des bénéficiaires de l'aide sociale. Cet instrument cristallise (Halpern et al., 2014) des orientations politiques et morales et véhicule une définition de ce que doit être le «public » cible des aides sociales : ce dernier ne doit pas dilapider l'argent public ni prévilégier une culture du « tout m'est 
dû » mais être guidé dans ses choix, tout en ayant la possibilité de recourir à des prestataires privés mis en concurrence.

Dans le cas français, le chèque ne suscite aucune analyse théorique ni idéologique de ce type. Et pourtant, les autorités publiques, essentiellement locales, ont toujours et encore recours à cet instrument. La généralisation du chèque et son utilisation s'inscrivent, en fait, dans un contexte général de réformes des EtatsProvidence. II s'agit de favoriser l'individualisation des aides sociales, (tendance qui apparait dès les années 1970 et se renforce dans les années 1990 et 2000) et de conduire leur mise en œuvre à un niveau plus local par des démarches adaptées aux divers contextes territoriaux (Esping-Andersen, 2008) ${ }^{2}$. La mise en place de dispositifs de contrôle de l'accès à ces aides (pièces justificatives demandées) se multiplient. Les contrôles d'effectivité sont désormais systématiques. Dès lors, l'usage du chèque apparait comme une politique rationnelle, cohérente puisqu'il 'est est utilisable que pour un bien précis ou le service auquel il est destiné. Son caractère étant le plus souvent nominal évite de verser des sommes indues, difficiles à récupérer par la suite.

Notre thèse est la suivante : si la diffusion du chèque en France a pu se faire aussi rapidement, c'est parce qu'il a perdu sa dimension idéoloqique et politique. II a été « dépolitisé » par les entreprises, les prestataires de service le commercialisant. Ainsi, le chèque est un instrument paradoxal: alors qu'il est porteur d'une vision néolibérale des politiques publiques et du public auquel il est destiné, il ne semble pas avoir été adopté pour ces mêmes raisons. Ainsi nous tenterons d'apporter une réponse à la question suivante : “comment cet instrument d'origine néolibérale,

\footnotetext{
2 ESPING-ANDERSEN Gøsta, The Three Worlds of Welfare Capitalism, Princeton University Press, 1990GLENNERSTER Howard, LE GRAND Julian, Le développement des quasi-marchés dans la protection sociale, "Revue Française d'Economie », vol 10, 1995, pp 111-135
} 
anglo-saxonne, a-t-il pu se diffuser aussi rapidement en France et à travers ses territoires sans que cette greffe et ce transfert ne soit remarqués ? »

La problématique retenue a permis d'expliciter les mécanismes qui font du chèque un vecteur implicite, caché, de l'extension d'une vision néolibérale et confère une influence déterminante au secteur privé, au point de modifier subrepticement les perceptions et les contenus politiques sous-jacents et de les rendre, in fine, plus acceptables.

Notre propos s'étaye sur une recherche conduite dans le cadre d'une thèse de doctorat (Lacheret, 2014) étudiant l'adoption du chèque, sa réinterprétation et sa mise en oeuvre par les collectivités territoriales françaises. Nous avons suivi l'extension du chèque de la Région Rhône-Alpes, pionnière en la matière, et sa transplantation vers d'autres régions (PACA) et conseils départementaux (Saône et Loire, Drôme) qui ont voulu dupliquer le dispositif et ses modalités de mise en œuvre. Au total, 45 entretiens ont été menés auprès d'élus, de cadres des collectivités et d'agents opérationnels, ainsi qu'auprès des acteurs travaillant dans les entreprises commercialisant les solutions-chèque pour déterminer leur perception quant à l'opérationnalité de l'instrument. Nous avons pu bénéficier d'un accès aux documents internes des collectivités et des entreprises commercialisant les chèques, ce qui nous a permis de mener une étude qualitative de trois terrains distincts. En Région Rhône-Alpes, premier lieu d'émergence du chèque-culture lancé par une collectivité locale française, il s'agissait d' offrir un accès à la culture et aux loisirs à une population ciblée. Sa diffusion rapide entre les collectivités françaises illustre bien le phénomène de dialogue et de parangonnage entre territoires. Les résultats de l'enquête montrent que les acteurs, à chaque transfert et importation de la méthode, se sont parfaitement approprié l'argumentaire néolibéral 
implicite du chèque, quelle que soit l'aide individuelle versée. Enfin, l'intervention des entreprises émettrices de chèques a été décisive pour ce qui est de l'introduction de logiques pragmatiques faisant de cet objet un dispositif dépolitisé beaucoup plus facilement acceptable du fait de son apparente neutralité politique.

\section{1- La dynamique de diffusion et d'appropriation du chèque en France}

Les premiers chèques permettant de verser une aide publique (les chèques vacances ou les titres de restauration étant destinés à être versés par les employeurs, nous les avons volontairement écartés de l'étude car ils ne sont pas de même nature alors qu'ils ont été introduits antérieurement) sont mis en place dans les années 1990 à l'échelle nationale, essentiellement dans le cadre des prestations sociales. On citera le «chèque emploi service universel » ${ }^{3}$ et le «chèque d'accompagnement personnalisé ${ }^{4}$. Mais c'est bien au sein des collectivités locales que s'organisent les premiers recours à cet outil.

En 1993, le conseil régional Rhône-Alpes crée le « chèque culture ». Destiné aux lycéens, ce carnet de quatre chèques permet, en échange d'une participation de 10 francs, de bénéficier d'un bon d'achat pour un livre, un spectacle vivant, une entrée dans un musée et une place de cinéma. Ce type d'aide se diffuse peu à peu : 21 régions sur 22 disposeront dans la décennie qui suit d'un système d'émission de chèques du même type. L'expérience ouvre la voie à l'émission de chèques formation (Poitou-Charentes), de chèque de mobilité (Puy de Dôme, Nord Pas de Calais Var), de chèque-santé (Conseil général de la Réunion), de chèques visant à l'acquisition de sièges-auto pour les bébés (Bouches du Rhône, Pyrénées

\footnotetext{
${ }^{3}$ Loi contre les exclusions du 29 juillet 1998

${ }^{4}$ Décret 99-862 du 6 octobre 1990
} 
Atlantiques), ou à l'achat de chauffe-eau solaires ou de chaudières à bois (plus de la moitié des Régions françaises).

Il existe donc deux types de chèques qui, bien que servant apparemment à la même chose (subvention pour l'acquisition par un public ciblé d'un bien ou d'un service) se distinguent par leurs visées respectives :

- Les chèques sociaux qui visent au ciblage et à la rationalisation de l'aide sociale.

- Les chèques permettant de verser des aides facultatives à un public qui n'a pas forcément de difficultés sociales mais aussi de remplacer une subvention tout en rendant tangible l'aide de la collectivité.

Présenté comme « un outil de politique culturelle pour la région Rhône-Alpes, qui le présente d'ailleurs comme l'axe prioritaire de sa politique en faveur de la " formation et de l'accès des jeunes à la culture »(Lacerenza, 2001) ${ }^{5}$, le chèque culture Rhône-Alpes a subi plusieurs transformations depuis son lancement en 1994 jusqu'aux derniers développements de la carte à puce (appelée carte M'RA) en 2013. D'un produit purement culturel destiné aux lycéens rhônalpins, il est ainsi devenu une carte « de l'autonomie » regroupant des services allant bien au-delà de la culture (sport, contraception, équipements professionnels des apprentis...) Le chèque culture peut donc être identifié comme un outil présentant une offre et permettant un choix individuel. II s'oppose dans son principe opérationnel à la politique culturelle plus habituelle de soutien à la demande.

\footnotetext{
${ }^{5}$ LACERENZA Sabine, "L'impensé des études sur les effets des politiques de tarification - L'exemple du chèque culture en région Rhône-Alpes ", in Olivier Donnat, Sylvie Octobre (dir.), Les publics des équipements culturels Méthodes et résultats d'enquêtes, Ministère de la Culture et de la Communication, Département des Études et de la Prospective, collection Les Travaux du DEP, 2001, p.170
} 
On retrouve cette idée dans les arguments avancés respectivement par Hervé Mariton, vice-Président en charge de la culture en 1998 où Amaury Nardone, Président de la Commission culture de l'époque ${ }^{6}$, lorsqu'ils parlent d'un « clivage » politique conditionnant la perception qu'ont les élus du chèque culture :

«(...) donc sur un élément comme le chèque culture, on a un exemple de clivage, qui est assez volontiers un clivage politique : les gens de droite ayant envie d'encourager une pratique individuelle, qui peut être une pratique éclairée, qui peut être une pratique bénéficiant d'un environnement, d'une pédagogie, mais qui à un moment, constate un choix individuel $»^{7}$

Ces acteurs politiques partagent la même vision du chèque comme étant un instrument du libre-choix permettant d'encadrer indirectement l'usage de la dépense publique tout en contraignant, par ailleurs, son bénéficiaire à le dépenser pour un usage bien précis.

II est donc surprenant de constater que l'alternance politique au sein du conseil régional ne freinera pas l'usage du chèque, pas plus que dans l'ensemble des régions. Les élections de 1998, puis celles de 2004, vont conduire à une alternance politique à gauche dans les conseils régionaux, les chèques inspirés de l'expérience rhônalpine se diffusent : chéquier «Tick'Art » (lle de France, 2001), chéquier " Ciné-Lecture » (Provence-Alpes-Côte-D’azur, 2002)... Les départements leur emboitent le pas : le « chèque culture » du Var est créé en 1999. II est le premier d'une série de chéquiers départementaux calqués sur le modèle rhônalpin.

\footnotetext{
${ }^{6}$ Entretien avec Amaury Nardone, Charbonnières les Bains, $1^{\text {er }}$ mars 1999

${ }^{7}$ Entretien avec Hervé Mariton, Crest, 15 avril 2000
} 
Cette recrudescence de chèques ciblant des aides facultatives va faire l'objet d'un recensement par le Ministère. Ainsi, François Rouet établit une cartographie des chèques par collectivité locale (Rouet, 2009).

Figure 1 : Collectivités disposant d'un dispositif de type « chèque culture » en 2009

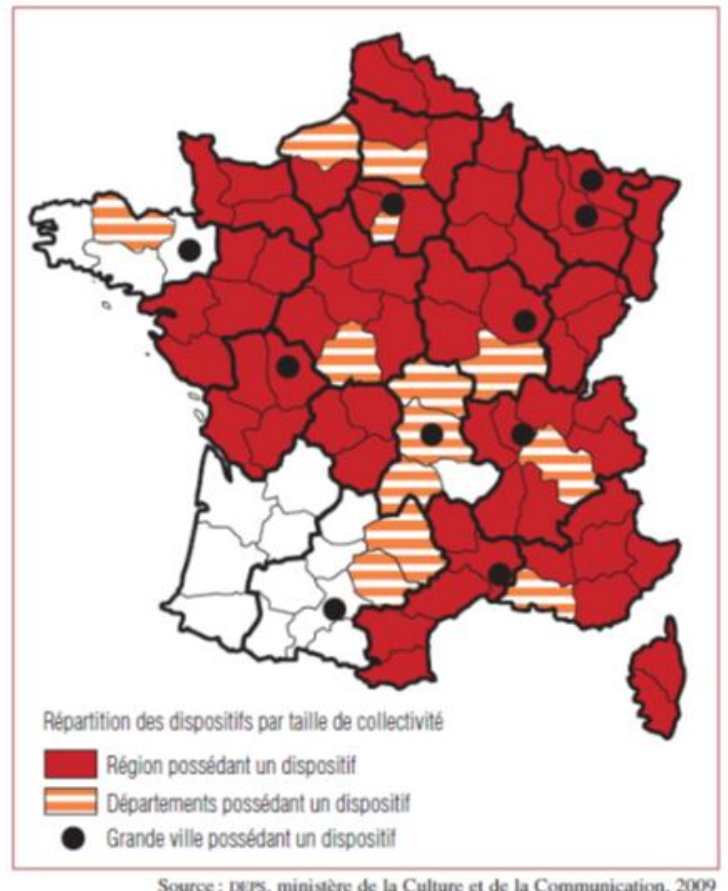

Parallèlement, d'autres dispositifs élargissent le champ d'action des politiques culturelles : des chèques sport proposent notamment une participation financière pour l'acquisition d'une licence sportive (Midi-Pyrénées, Limousin), ou des chèques « mixtes » à destination des collégiens (Drôme, Allier.

L'exemple du chèque culture, du fait de son image positive et de sa popularité permet de comprendre l'absence de clivage politique à son endroit. En effet, la filiation libérale n'est pas véritablement lisible ni visible ou revendiquée par les acteurs. C'est ainsi que la diffusion du chèque au sein des collectivités de tout bord se fait, par conséquent, de façon consensuelle. 
La restitution de la trajectoire du chèque culture et énergie pose alors une question : peut-on vraiment considérer que leur circulation s'apparente à la diffusion d'une vision « néolibérale » du public des politiques publiques ?

\section{Le chèque est-il un instrument néolibéral caché ?}

\subsection{Des arguments fragmentés et divers}

Les entretiens menés nous permettent de comparer les arguments justifiant la mise en place du chèque par les acteurs des collectivités françaises et ceux utilisés par les différents auteurs anglo-saxons dans les années 1990 et 2000 que nous avons présentés en introduction.

Or, il s'avère que ces acteurs ne reprennent pas l'ensemble de la doxa néolibérale soutenant l'utilisation du chèque, telle que nous l'avons décrite plus haut: toutefois, ils se réfèrent systématiquement à au moins l'un des arguments avancés par cette même doxa. Chaque acteur mobilise la dimension qui l'intéresse le plus dans l'ensemble de cet argumentaire sans afficher une adhésion explicite à ce discours. Certains acteurs vont ainsi valoriser l'argument $\mathrm{du}$ « libre-choix $»^{8}$, d'autres insistent davantage sur le fait que le chèque est un moyen de faire accepter une aide fléchée à la population selon un double principe fondé sur le choix et l'équité au sens de la définition de Steuerle ${ }^{9}$. L'argument d'une mise en compétition des fournisseurs est également convoqué dans les entretiens ${ }^{10}$. Le chèque peut également être présenté par les acteurs comme un outil servant à remplacer un autre programme

\footnotetext{
${ }^{8}$ Entretien avec Amaury Nardone, Lyon, 10 février 2010

${ }^{9}$ Entretien avec Joël Crémilleux, DGS du CG de la Drôme, Valence, 11 juillet 2011

${ }^{10}$ Entretien avec Nicolas Gomord, chef du service sport jeunesse, CG de la Drôme, Valence, 11 juillet 2011
} 
d'aide $^{11}$. La limitation implicite du choix des bénéficiaires est également citée, notamment par l'ancien Directeur Général adjoint des Services au Conseil général de Saône et Loire $^{12}$ à propos du chèque permettant de verser la Prestation de Compensation du Handicap. Enfin, l'argument financier selon lequel le versement d'une aide sous la forme d'un chèque serait une source d'économies pour les collectivités locales compte également parmi les arguments les plus développés par les acteurs du chèque social. ${ }^{13}$

Deux autres arguments plus pragmatiques, et donc moins directement liés à la doxa néolibérale, reviennent dans les entretiens : la fonction persuasive d'une action de communication et la réduction des coûts de transactions, c'est-à-dire le fait de matérialiser une aide sociale, de la rendre concrète pour celui qui la reçoit et d'en simplifier la gestion administrative lors de l'instruction des dossiers qui sont externalisés à un opérateur privé. On peine, dès lors, à identifier précisément la figure du destinataire et des «publics » qui y sont associés par les acteurs de la mise en place du chèque.

\subsection{Le «public » composite du chèque : une cible multiple qui diffère selon les secteurs}

Les acteurs interrogés donnent moins un sens politique au chèque qu'ils ne valorisent son utilité dans le cadre de l'aide allouée. Le chèque correspond à la définition très large que lui donne Harvey Rosen (Rosen, 2001). Les acteurs contemporains, tout en reprenant des fragments de discours inspirés du modèle néolibéral, promeuvent donc in fine une forme dépolitisée du chèque liée à la

\footnotetext{
${ }^{11}$ Entretien avec Brigitte Ducourtil, chef du service énergies, Région Rhône-Alpes, Lyon, 28 avril 2013

12 Entretien avec Pierre Bucco, Vesoul, 31 janvier 2013 Est-ce utile?

${ }^{13}$ Boris Vallaud, op. cit.
} 
neutralité de son objectif pragmatique qui semble rendre ainsi possible sa diffusion dans des collectivités gérées majoritairement par la gauche. Le chèque ne matérialise pas les intentions politiques qu'un instrument trop ouvertement politisé pourrait afficher. II devient un objet de compromis.

L'un des intérêts de l'étude a été d'interroger les acteurs sur l'utilité qu'ils voyaient au chèque et donc, en creux, comment ils en concevaient les usagers. II est apparu une différence de conception notable entre deux types de représentation que se faisaient les récipiendaires d' des aides facultatives comme celles du« chèque culture », et les aides plus ouvertement sociales des chèques d'aide à la personne par exemple.

Dans le cas des aides sociales auxquelles «public » a droit, ce dernier est construit comme un ensemble de personnes dont la rationalité reste très limitée et qui fait, de ce fait, l'objet d'une certaine méfiance paternaliste de la part des élus comme des cadres administratif ou des agents opérationnels. ${ }^{14}$

Le public est supposé ne pas être assez « mûr » - un cadre administratif d'un Conseil général évoque des «personnes qui ne comprennent pas tout » - et ne sont pas en mesure d'utiliser à bon escient l'allocation. Contraindre la dépense et flécher son objet est donc considéré comme un moyen d'encadrer et d'aider effectivement les bénéficiaires selon des normes restrictives établies par le destinateur. ${ }^{15}$

A l'inverse, le public ciblé par les chèques et qui porte sur d'autres secteurs d'action publique est décrit comme moins « immature », plus responsable. Il est donc capable d'initiative et «naturellement» plus demandeur de liberté de choix importante. Cette différence d'attitude institutionnelle ne s'appuie pas sur la nature

\footnotetext{
${ }^{14}$ Entretiens notamment avec Boris Vallaud, op. cit et Amaury Nardone, op. cit.

${ }^{15}$ Entretien avec Pierre Bucco, op. cit.
} 
intrinsèque des chèques. En effet, même si, dans les faits, le chèque social ouvre une série de choix pour son bénéficiaire, cette dimension d'autonomie du choix n'est jamais évoquée par les acteurs. On retrouve par contre mention de la notion delibre choix au premier rang des arguments présents dans les entretiens lorsque les acteurs évoquent les chèques permettant de verser des aides facultatives.

Ainsi, le public visé par les aides facultatives est mis en position d'excercer sa liberté de choix et et de faire jouer le marché privé au moyen du dit chèque. Cette conception libérale des sujets acteurs (empowered) qu'implique ce type d'instrument pourrait réactiver un clivage plus politique droite/gauche au sein des institutions régionales concernées, comme on a pu le constater avec l'introduction du chèque culture en Rhône-Alpes. ${ }^{16}$

Pourtant, ce clivage ne se lit pas véritablement dans les comptes-rendus d'entretien. A droite comme à gauche, les élus décrivent le chèque comme un instrument « valorisant » les compétences et les facultés du public ciblé et offrant des marges de manœuvre et de liberté dont il saura se servir à bon escient.

Cette perception positive est partagée par les acteurs administratifs qui voit dans le public bénéficiaire des aides facultatives des acteurs responsables et autonomes dans leurs décisions. Le directeur du service jeunesse et sport de RhôneAlpes parle d'« une carte qui peut avoir vocation à tendre vers une carte de l'autonomie du jeune, c'est-à-dire qui peut servir comme on a commencé à le faire à ce que le jeune soit acteur de son parcours d'autonomie $"{ }^{17}$. Son collègue, directeur de la carte M'RA, parle «d'un acte d'autonomisation du jeune, vraiment pour le

\footnotetext{
${ }^{16}$ Entretien avec Charles Millon, op. cit.

${ }^{17}$ Entretien avec Thomas Senn, Lyon, 20 avril 2013
} 
laisser libre de choisir $»^{18}$. La chef du service jeunesse de la Région PACA décrit le chèque comme " une aide directe au jeune pour son ouverture à la culture, une aide très individuelle ${ }^{19}$. Dans la Drôme, la responsable du chéquier destiné aux collégiens en fait un outil "d'implication du jeune dans la vie associative ${ }^{20}$, et une façon de le responsabiliser et lui apprendre à gérer par lui-même le subside.

Ainsi, le chèque ne fait pas que matérialiser à une subvention monétaire expost, il est également porteur de représentations ex-ante non seulement de l'aide mais aussi des public visés. Un même objet véhicule donc une représentation différente du public qu'il est censé aider, au-delà de toute référence à une réalité concrète.

Dans les deux cas de figure, un autre «public » du chèque est également convoqué : le «grand public » ou «l'opinion publique ». De nombreux acteurs avancent l'idée selon laquelle l'affichage ex-ante d'un contrôle de l'utilisation accroit la légitimité externe des aides sociales et facilite le bien-fondé de leur octroi , notamment auprès des personnes à qui elles ne s'adressent pas. Le DGS de la Drôme indique que «les gens sont pour qu'on contrôle la dépense des bénéficiaires ${ }^{21}$. Son homologue de Saône et Loire reprend quasiment la même phrase : "sur le chèque lui-même il n'y a pas eu vraiment de débat, les gens ont trouvé ça plutôt pas mal de pouvoir avoir de la visibilité et du contrôle $»^{22}$.

Cette affirmation est particulièrement récurrente chez les acteurs politiques comme Amaury Nardone ${ }^{23}$, Charles Millon ou le socialiste Pierre Martinerie, vice-Président du Conseil général de Saône et Loire, qui insistent sur les gages à donner à la

\footnotetext{
${ }^{18}$ Entretien avec Patrick Darnaud, Lyon, 8 octobre 2012

${ }^{19}$ Entretien avec Jocelyne Giamportone, Marseille, 11 février 2010

${ }^{20}$ Entretien avec Stéphanie Loullier, Valence, 23 juin 2010

${ }^{21}$ Entretien avec Joël Crémilleux, op. cit.

${ }^{22}$ Entretien avec Boris Vallaud, op. cit.

${ }^{23}$ Entretien avec Amaury Nardone, op. cit.
} 
population, sur le « sérieux » que doit représenter l'attribution de l'aide publique et de l'argent du contribuable.

Les entretiens révèlent donc le caractère pluriel et polysémique des argumentaires légitimant le recours aux chèques. S'ils renvoient bien à des fondements idéologiques inspirés d'une conception néolibérale de l'action publique, ils ne s'enracinent pas dans des croyances politiques identifiables. En réalité, leur mode de justification est à chercher hors des paradigmes intellectuels et du seul champ politiques mais dans la logique performative de l'instrument. Les arguments reprennent plutôt les vocables et formulations des entreprises commercialisant les chèques, des organismes en charge des procédures et modes de versement des aidesqui ont su imposer leur langage commercial aux clients et aux acteurs institutionnels à qui elles fournissent les titres et les procédures.

\section{Les entreprises médiatrices : vecteur et acteurs de dépolitisation d'une « cause sans adversaire »}

Les acteurs rencontrés l'affirment à tous les niveaux : la gestion d'un système de chèques ou de cartes est complexe. C'est donc auprès des entreprises spécialisées que les acteurs publics vont chercher l'expertise technique nécessaire en externalisant leur besoins. S'il est difficile de connaitre le nombre de chèques gérés par des prestataires privés parmi les 43 dispositifs recensés en 2009 par l'étude du Ministère de la culture, les auteurs sont conscients que «Lorsque la prestation est déléguée, les grands prestataires de services [...] peuvent s'appuyer sur leurs réseaux préexistants ${ }^{24}$. Ils assument la mise en œuvre technique des chèques mais aussi les coûts de transaction monétaires et communicationnels liés à

\footnotetext{
${ }^{24}$ Op.cit
} 
leur émission tout en euphémisant la dimension politicienne des politiques publiques qui en ont été le préalable. Les ordonnateurs institutionnels peuvent ainsi externaliser les coûts techniques et politiques de leur gestion.

\subsection{De la prestation de service au lobbying politique?}

Les entreprises émettrices de titres de restauration, par exemple, mettent ainsi à disposition des ordonnateurs publics une expertise et des produits issus d'autres types de prestations destinées également au grand public et qui utilisent des titres monétaires comme les «chèques ». Les marchés publics de fournitures de chèques aux agents des collectivités territoriales sont âprement disputés par les quatre émetteurs présents en France : Edenred (Ticket Restaurant), le Groupe Up (Chèque Déjeuner), Sodexo CCR (Chèque Restaurant) et Natixis Intertitres (Chèque de Table). Compte-tenu de la maturité du marché des chèques, et pour profiter des opportunités légales et fiscales existantes, ces entreprises émettrices de chèque ont créé d'autres types de produits pour élargir la palette de leur offre. Ils sont assez semblables en termes de modèle économique (chèques cadeaux, CESU...) mais permettent de créer de nouveaux marchés offreurs.

En plus de ces gammes de chèques classiques, certains émetteurs ont créé des titres plus spécifiques, établis sur mesure pour les collectivités désireuses de mettre en place une aide élargie à des publics plus segmentés . Le Groupe Up développe, à partir de 1999, un service d'ingénierie de chèques "sur mesure » capable de s'adapter à la demande des collectivités (nommée " adéquation »). Chez Edenred, le principe fut le même avec la création de la branche « Ticket à la carte » à la même période. 
Cette réponse ciblée aux demandes des collectivités a correspondu, à la fin des années 1990, à l'essor de nouveaux dispositifs culturels qui ont privilégié l'usage du chèque.

Les produits «sur mesure » sont donc moins des sources de revenus accrus pour les émetteurs que des outils de communication et d'influence permettant de valoriser les entreprises et leurs marques sur un marché très concurrentiel, dont les produits les plus rentables sont, par ailleurs, strictement identiques ${ }^{25}$.

II est donc logique que les trois plus importants émetteurs aient profité de l'opportunité représentée par le versement des aides à la population pour s'engager dans la production de chèques sur mesure. C'est le secteur culturel dans un premier temps qui a été le premier bénéficiaire de cette nouvelle manne, puis, au gré de la diffusion et de l'évolution des demandes des collectivités, les nouveaux marchés se sont également portés vers des domaines comme l'environnement, les transports et l'action sociale.Les entreprises prestataires ont dû réorganiser leurs services et créer des départements entiers dédiés à l'ingénierie des collectivités, capables de répondre à des appels d'offres complexes et d'assurer la gestion de ces dispositifs.

Cette évolution n'est pas sans conséquences pour les entreprises et les institutions publiques. Ainsi, le Groupe Chèque Déjeuner, , a créé en 2006, en plus d'adéquation, un service commercial spécialisé -le Département des relations institutionnelles - visant à promouvoir son ingénierie «sur mesure » auprès des collectivités .

Cette montée en puissance de la demande de chèques culturels des collectivités coïncide parfaitement avec les débuts de la diffusion des « chèques cultures » au sein de Départements et de Régions. L'entreprise va donc

\footnotetext{
${ }^{25}$ Entretien notamment avec Stéphane Lefebvre, Epernay, 21 décembre 2012
} 
accompagner cette nouvelle demande, puis, de plus en plus, la susciter par une démarche qui s'apparente à une forme de lobbying et de stratégie d'influence.

Parallèlement à cette réponse technique du secteur privé et face à l'augmentation de la demande de dispositifs par chèque que les collectivités pensaient ne pas savoir gérer, une organisation plus commerciale a vu le jour afin de vendre et promouvoir des dispositifs sur mesure. Ils modifient souvent le schéma de fonctionnement des l' organisations. Les entreprises ont dû, pour cela, réviser leurs méthodes de vente : il ne s'agit désormais plus de vendre un produit existant à une collectivité en répondant à un besoin précis mais de susciter l'envie, d’identifier les acteurs clés, d'être une ressource technique lors de la rédaction d'un éventuel cahier des charges pour, finalement, de faire effectuer le travail opérationnel par la filiale « adéquation », en charge de la gestion de dispositifs sur mesure.

Ce changement de stratégie a par exemple été mené très rapidement, en quelques mois par la Direction générale du groupe Up (chèque déjeuner). Cette adaptation à la nouvelle configuration témoigne de la volonté d'influer davantage sur les décisions des collectivités locales en les incitant à adopter le chèque pour toute sorte d'aide individuelle obligatoire ou non. Deux autres prestataires, Accor services (qui deviendra Edenred) et Sodexo CCR opèreront en parallèle des mutations similaires.

\subsection{L'argumentation " commerciale " du chèque : un discours public/privé co- produit ?}

Cette démarche marketing, se double d'un lobbying efficace. Elle a eu pour conséquence de généraliser la promotion d'un discours argumentatif soulignant la 
dimension éthique et de responsabilité sociale qu'offre une relation d'aide et revendiquant ainsi l' efficacité pratique mais aussi la « neutralité politique » de la démarche. Le chèque et sa promotion deviennent une «cause sans adversaire » (Cloteau, Mourad, 2016, Juhem, 2001) mais aussi plus indirectement une œuvre sociale co-produite.

L'action commerciale des entreprises conceptrices de chèques auprès des collectivités apparaît ainsi comme un des facteurs expliquant la dynamique d'appropriation, et de diffusion accélérée des chèques au sein des collectivités françaises. Si l'on se fie à l'argumentaire de vente des émetteurs qui les commercialisent auprès des collectivités, le passage au chèque permet une «garantie de bonne utilisation des fonds affectés», une «gestion simplifiée du dispositif d'aide ", et « son caractère anonyme est une source d'une plus grande dignité, d'une liberté de choix, d'une prise de contact avec les acteurs de la vie locale $»^{26}$.

En 2010, dans deux courriers destinés aux directeurs des services «jeunesse » des collectivités ainsi qu'aux élus en charge de cette thématique, les arguments développés sont ceux de la simplification administrative, du contrôle de la dépense publique et de la lisibilité 27

Ce sont donc les entreprises commercialisant les dispositifs qui ont joué ce rôle central de médiation en privatisant et en transformant les modalités de versement des aides sociales via les chèques. Elles ont pu implanter une représentation de l'aide individuelle a priori complètement fonctionnelle mais aussi légitimer un modèle mixte de partenariat privé/public. Prendre en compte l'influence

\footnotetext{
${ }^{26}$ On retrouve ces argumentaires sur les plaquettes promotionnelles et les sites internet des principaux émetteurs.

${ }^{27}$ Source interne au Groupe Chèque Déjeuner
} 
cognitive de ces discours d'action publics et privés permet d'expliquer le degré de fragmentation et de bricolage des argumentaires politiques mobilisés tant par les acteurs politiques et administratifs lors de la mise en place des chèques que par les groupes privés désireux d'obtenir de nouveaux marchés et de susciter une relation de confiance et de partenariat avec des instiutions publiques. L'objectif de leur usage ne peut donc se réduire à la seule volonté de conformer le public à une injonction néolibérale, quand bien même le caractère néolibéral de ces instruments et la prégnance diffuse d'un modèle néolibéral peuvent être, par ailleurs, attestés.

\section{Conclusion}

Il faut donc s'interroger sur les raisons objectives du transfert, de l'adoption et de la circulation d'un outil, le chèque/voucher, présenté comme l'émanation d'un paradigme néolibéral d'origine anglo-saxonne. Cette diffusion au sein des collectivités locales françaises s'est effectuée de façon transversale, sans intervention particulière ou directe de l'Etat comme ce fut le cas pour d'autres dispositifs et sans qu'il n'y ait eu de débat particulier.

Au vu des origines très « libérales » du chèque revendiquées par un certain nombre d'hommes politiques français l'ayant introduit dans les années 90, on aurait pu penser que les discours véhiculés par les parties prenantes du chèque soient particulièrement tranchés, au point qu'un véritable débat politique s'instaurât dans les collectivités où il est implanté. C'est très loin d'être le cas puisque le chèque est devenu une « cause sans adversaire », un instrument technique jugé utile et pratique et dont les caractéristiques marchandes et instrumentales ne dérangent pas les acteurs interrogés. 
La segmentation du public ciblé semble avoir conditionné les modes et les styles d'appropriation des différents types de chèque. Lorsque le chèque visait le versement d'une aide sociale, l'encadrement des pratiques était prescrit ex-ante, Lorsque le chèque permettait de verser une aide culturelle, c'était quasiment l'argumentaire inverse qui était développé .

L'adoption de cet instrument néolibéral en France a donc largement reposé sur les argumentaires commerciaux développés par les entreprises émettrices déjà implantées et qui parfois, fournissaient déjà les collectivités. Elles se sont structurées pour répondre à ce besoin nouveau au niveau local, le faisant souvent découvrir aux collectivités en le présentant comme un outil de communication et de simplification administrative. Les élus et les agents des collectivités se sont emparés du chèque pour répondre à des problématiques concrètes tout en reprenant partiellement la rhétorique néolibérale que l'on retrouve chez les chercheurs anglo-saxons mais c'est ce renfort, cette «assistance argumentative» développée par les entreprises émettrices qui a permis de faire en sorte que l'instrument-chèque se diffuse.

Les chèques sont toutefois porteurs d'une représentation de l'aide sociale et de ses bénéficiaires, ils sont finalement le vecteur d'un changement de conception des aides sociales et de leurs publics et accompagnent le processus de passage d'une logique universaliste à une logique de ciblage des aides individuelles, désormais très avancée.

En effet, cette arrivée du chèque dans les collectivités locales françaises, à l'orée des année 90, est concomitante avec l'introduction massive des outils de gestion du «nouveau management public » et de leur transfert et appropriation par les institutions publiques. L'originalité de cet instrument en particulier réside donc principalement dans son mode de diffusion et « l'accompagnement argumentatif » 
dont ont fait preuve les entreprises et les institutions publiques pour introduire une logique utilitariste accompagnée d'une représentation ciblée du public bénéficiaire de ces chèques.

La promotion de nouveaux outils de gouvernance est donc le produit d'une époque, d'une histoire institutionnelle et de la recherche discursive d'une cohérence entre logiques publiques et privée plus que d'une seule optimalité de fonctionnement. En se retrouvant autour de représentations et d'objets de compromis communs comme le chèque, les acteurs faisaient indirectement l'économie de luttes et de débats plus politiques. La neutralité apparente du recours à la rationalité instrumentale et à sa légitimité apolitique a permis le développement d'une dynamique normative partagée en totale compatibilité avec la montée du nouveau management public, de nouveaux modes d'appropriation publics partagés. La prégnance d'un esprit, de valeurs, de représentations communes qui évacuent le conflit et le débat et privilégient la coproduction d'innovations institutionnelles a fait le reste.

\section{Biographie}

Arnaud Lacheret est Docteur en Science Politique, chercheur associé au Laboratoire Pacte - Sciences Po Grenoble et enseignant-chercheur à l'Idrac Business School. 